\title{
Macroscopic Findings Specimen Condition
}

National Cancer Institute

\section{Source}

National Cancer Institute. Macroscopic Findings Specimen Condition. NCI Thesaurus.

Code C119869.

A characteristic that refers to the physical and organoleptic state of a biospecimen used for a macroscopic findings assessment. 